\title{
Research on the Graft Copolymerization of EH-lignin with acrylamide
}

\author{
Run Fang ${ }^{1,2}$, Xian-Su Cheng ${ }^{* 1}$, Jian Fu' ${ }^{1}$, Zuan-Bin Zheng ${ }^{1}$ \\ ${ }^{1}$ College of Material Science and Engineering, Fuzhou University, Fuzhou 350002, China; ${ }^{2}$ Department of Chemistry and Chemical \\ Engineering, Minjiang University, Fuzhou 350011, China. \\ *Corresponding author: chengxiansu@,fzu.edu.cn
}

Received 13 April 2009; revised 14 May 2009; accepted 23 May 2009.

\begin{abstract}
Lignin isolated from enzymatic hydrolyzed cornstalks (EH-lignin) is a renewable natural polymer noted for its versatility and applicability in a variety of uses. Graft copolymerization of EH-lignin with acrylamide (AM) and the application of this copolymer as a flocculant in dye wastewater treatment were studied in this article. The influences of some factors on yield of copolymer and the grafting ratio were investigated and the structure of EH-lignin/AM graft copolymer was characterized by FT-IR. According to the yield and the grafting ratio, the optimum conditions for graft copolymerization were as follows: initiator $\mathrm{K}_{2} \mathrm{~S}_{2} \mathrm{O}_{8}-\mathrm{Na}_{2} \mathrm{~S}_{2} \mathrm{O}_{3}$ with a quantity $3 \mathrm{wt} \%$ of EH-lignin, mass ratio of $A M$ to EH-lignin was 2 3, reaction time $4 \mathrm{~h}$ and temperature at $50^{\circ} \mathrm{C}$. It was found that the absorption capacity of graft copolymer to two azo-dyes was enhanced with the increase of grafting ratio. Furthermore, the residue concentration of EH-lignin/AM graft copolymer remained in the supernatant after flocculation was much lower than that of pure EH-lignin.
\end{abstract}

Keywords: Lignin; Acrylamide; Graft Copolymerization; Dye Wastewater; Decoloration

\section{INTRODUCTION}

At present, the fossil resources are rapidly running out and the environmental pollutions are getting even more serious throughout the world. Great attention has been paid to the development of sustainable technologies based on renewable raw materials $[1,2,3,4]$. As a natural polymer, lignin is a renewable and biodegradable resource and noted for its versatility and applicability in a variety of uses. Making use of these biomaterials will not only enhance the economic benefit of bioengineering but also diminish environmental pollutions [5,6].

EH-lignin is a novel ornanosolv lignin isolated from the residue of enzymatically hydrolyzed cornstalks as a by-product of fuel ethanol industry [7]. Compared with traditional lignosulfonate or alkali lignin, EH-lignin possesses some valuable characteristics: lower content of sugar, less impurities and narrow molecular weight distribution. Furthermore, since the enzymatic hydrolysis process of the cornstalks is carried out under relatively mild conditions, many functional groups such as phenolic hydroxyl, alcoholic hydroxyl and methoxyl are well preserved in EH-lignin $[8,9,10]$.

Due to its abundant functional groups, EH-lignin can be used in dye wastewater treatment by adsorbing dyes through hydrogen bonding under acidic conditions. However, the concentration of residue lignin remains in supernatant after flocculation is very high, which may leads to a secondary pollution. In order to minimize the potential secondary pollution, graft copolymerization of EH-lignin with acrylamide (AM) was studied in this paper. The effects of some factors on the copolymerization were investigated. The structure of EH-lignin/AM copolymer was analyzed by FT-IR and its application in the dye wastewater treatment was evaluated. The residue concentration of this flocculant remains in the supernatant after flocculation was measured.

\section{EXPERIMENTAL}

\subsection{Materials}

EH-lignin was supplied by Tianguang fuel ethanol company (He'nan, China) in powder form and purified in laboratory according to procedures described in our previous article [4]. Characteristics of purified EH-lignin are shown in Table 1. The details of two azo-dyes, acid red 274 (AR 274) and reactive red X-3B (RR X-3B), were shown in Fig. 1 and Table 2. Acrylamide were purchased from Guanghua chemical reagent Co., Ltd, China. All other reagents were of analytical grade.

Table 1. Characteristics of EH-lignin.

\begin{tabular}{cccccc}
\hline \multirow{2}{*}{ EHLignin } & $\begin{array}{c}\text { Residual } \\
\text { sugar/\% }\end{array}$ & $\begin{array}{c}\text { Ash } \\
/ \%\end{array}$ & $\begin{array}{c}\text { Phenolic hy- } \\
\text { droxyl/mmol.g }\end{array}$ & Mw & Mw/Mn \\
\cline { 2 - 6 } & 0.22 & 0.39 & 4.25 & 2062 & 1.22 \\
\hline
\end{tabular}




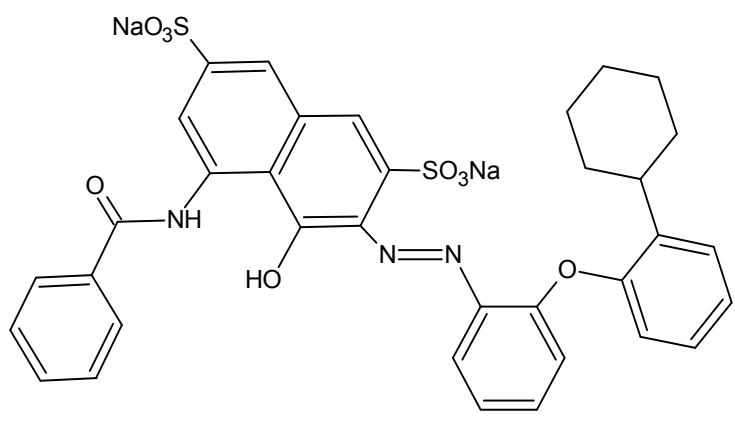

(a)

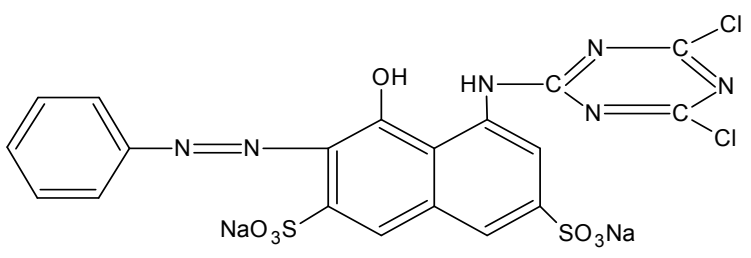

(b)

Figure 1. Molecular structure of (a) Acid red 274 and (b) Reactive red X-3B.

Table 2. Details of the dyes.

\begin{tabular}{lcccc}
\hline \multicolumn{1}{c}{ Dyes } & Abbreviation & Molecular formula & CAS number & $\lambda \max (\mathrm{nm})$ \\
\hline Acid red 274 & AR 274 & $\mathrm{C}_{35} \mathrm{H}_{31} \mathrm{~N}_{3} \mathrm{Na}_{2} \mathrm{O}_{9} \mathrm{~S}_{2}$ & $72828-83-2$ & 527 \\
Reactive red X-3B & RR X-3B & $\mathrm{C}_{19} \mathrm{H}_{10} \mathrm{Cl}_{2} \mathrm{~N}_{6} \mathrm{Na}_{2} \mathrm{O}_{7} \mathrm{~S}_{2}$ & $12226-03-8$ & 538 \\
\hline
\end{tabular}

\subsection{Synthesis and Characteristics of EH- lignin/ AM Graft Copolymer}

Graft copolymerization reactions were carried out in a jacketed reactor flask equipped with a stirrer and a reflex condenser under $\mathrm{N}_{2}$ protection. Appropriate amount of EH-lignin, AM and initiators were dissolved in $\mathrm{NaOH}$ aqueous solution and then reacted at different temperatures for a period of time. When a reaction was finished, copolymer product was precipitated by acidification and isolated in a centrifuge. In order to remove monomers, EH-lignin/AM graft copolymers were washed by distilled water and then vacuum dried.

Viscosity measurement of lignin/AM copolymer in water solution was conducted by an Ubbelohde type viscometer at $30.0 \pm 0.1^{\circ} \mathrm{C}$. Extrapolation procedure from data obtained for 5 concentrations of solutions was used to calculate $[\eta]$ from Huggins equation, $\eta_{\mathrm{sp}} / \mathrm{c}=[\eta]+\mathrm{k}$ $[\eta]^{2} \mathrm{c}$. The intrinsic viscosity was then used to evaluate the molecular weight of graft copolymers prepared with different initiators.

The chemical structure of graft copolymer was analyzed using FT-IR2000 spectrometer (Perkinelmer, U. S.) and the spectra were recorded in the range of 500-4000 $\mathrm{cm}^{-1}$.

Yield of EH-lignin/AM copolymer and the grafting ration were determined by Eq.1 and Eq.2 respectively.

Yield

$$
Y(\%)=\frac{W_{2}}{W_{0}+W_{1}} \times 100 \%
$$

Grafting ratio

$$
G R(\%)=\frac{W_{2}-W_{0}+W_{3}}{W_{0}-W_{3}} \times 100 \%
$$

where $\mathrm{W}_{0}$ is the weight of EH-lignin; $\mathrm{W}_{1}$ is the weight of $\mathrm{AM}$ monomer; $\mathrm{W}_{2}$ is the weight of the graft copolymer; $\mathrm{W}_{3}$ is the weight of lignin remained in the supernatant.

\subsection{Adsorption and Decoloration of AR 274 and RR X-3B Dye Wastewater}

The adsorption and decoloration of AR 274 and RR $\mathrm{X}-3 \mathrm{~B}$ dye wastewater by EH-lignin and EH-lignin/AM grafted copolymer was investigated by static adsorption method. Firstly, a certain amount of flocculant was weighed and dissolved in $2 \mathrm{ml} 1 \% \mathrm{NaOH}$ aqueous solution. Afterward, $200 \mathrm{ml}$ dye wastewater with a concentration of $500 \mathrm{mg} / \mathrm{L}$ was added into aforesaid copolymer solution, stirring $3 \mathrm{~min}$ to make sure the mixture wellmixed and then kept undisturbed for 1h. Finally, the solution was acidified with $\mathrm{HCl}$ to $\mathrm{pH}=4$ and then filtered after another $10 \mathrm{~min}$ standing. The concentrations of dye wastewater before and after treatment were measured at $\lambda \max$ mentioned in Table 2 by UV-2450 spectrophotometer. The total organic carbon (TOC) content of the supernatant was measured by TOC-V analyzer (Shima$\mathrm{dzu}$, Japan) to evaluate the residue amount of the flocculant and the dyes remained in the supernatant after flocculation. The absorption amount, decoloration rate and TOC removal can be calculated by Eq.3, Eq.4 and Eq.5 respectively.

Adsorption amount

$$
Q=\frac{\left(C_{0}-C\right) \times V}{W}
$$

Decoloration rate

$$
E=\frac{A_{0}-A}{A_{0}} \times 100 \%
$$

TOC removal rate 


$$
R=\frac{B_{0}-B}{B_{0}} \times 100 \%
$$

where $\mathrm{Q}$ is the adsorption amount, $\mathrm{mg} / \mathrm{g} ; \mathrm{C}_{0}$ and $\mathrm{C}$ are the concentrations of the dye solution before and after treatment, $\mathrm{mg} / \mathrm{L} ; \mathrm{V}$ is the volume of dye solution, $\mathrm{L} ; \mathrm{W}$ is the amount of graft copolymer, $\mathrm{g}$; $\mathrm{E}$ is decoloration rate; $\mathrm{A}_{0}$ and $\mathrm{A}$ are the absorbance of the dye solution before and after treatment; $\mathrm{R}$ is the TOC removal rate; $\mathrm{B}_{0}$ and $\mathrm{B}$ are the TOC values of the dye solution before and after treatment.

\section{RESULTS AND DISCUSSION}

The effects of various factors on the yield of copolymer and the grafting ratio were investigated to determine the optimum conditions for graft copolymerization. The basic reaction conditions of these experiments were as follows: the dosage of EH-lignin was $2.0 \mathrm{~g}$, acrylamide was $4.0 \mathrm{~g}, 100 \mathrm{~g} 1 \% \mathrm{NaOH}$ aqueous solution was used as solvent, the amount of $\mathrm{K}_{2} \mathrm{~S}_{2} \mathrm{O}_{8}-\mathrm{Na}_{2} \mathrm{~S}_{2} \mathrm{O}_{3}$ was $3 \%$ of the weight of EH-lignin, the reaction temperature was $50^{\circ} \mathrm{C}$ and the reaction time was $4 \mathrm{~h}$.

\subsection{Effects of Some Factors on Graft Copolymerization}

\subsubsection{Effects of Different Kinds of Initiators on Graft Copolymerization}

The graft copolymerization of EH-lignin with AM was carried out in aqueous solution, therefore six watersoluble radical initiators were chosen and their effect on grafted copolymerization was studied. The results were showed in Table 1 and the synthesis conditions were as follows: weight of lignin was $2.0 \mathrm{~g}$, acrylamide $4.0 \mathrm{~g}$, reaction temperature was $50^{\circ} \mathrm{C}$, reaction time was $4 \mathrm{~h}$ and the dosage of initiator was $3 \%$ of the weight of EH-lignin.

Since the raw materials and the reaction procedures are identical, the chemical structure of lignin/AM copolymers initiated by different initiators is quite similar to each other. Therefore, higher [ $\eta]$ of a lignin/AM copolymer's aqueous solution may indicates a larger molecular weight of this copolymer. It can be seen in Table 1

Table 3. Effects of different initiators on yield and intrinsic viscosity of the graft copolymers.

\begin{tabular}{lcc}
\hline \multicolumn{1}{r}{ Initiator } & Yield $/ \%$ & {$[\eta] / \mathrm{mL}^{-1}$} \\
\hline $\mathrm{Fe}^{2+}-\mathrm{H}_{2} \mathrm{O}_{2}$ & 34.30 & 35.76 \\
$\left(\mathrm{NH}_{4}\right)_{2} \mathrm{Ce}\left(\mathrm{NO}_{3}\right)_{6}$ & 30.86 & 34.51 \\
$\mathrm{~K}_{2} \mathrm{~S}_{2} \mathrm{O}_{8}$ & 37.83 & 35.37 \\
$\left(\mathrm{NH}_{4}\right) \mathrm{S}_{2} \mathrm{O}_{8}$ & 34.55 & 37.15 \\
$\mathrm{~K}_{2} \mathrm{~S}_{2} \mathrm{O}_{8}-\mathrm{NaHSO}_{3}$ & 37.40 & 36.93 \\
$\mathrm{~K}_{2} \mathrm{~S}_{2} \mathrm{O}_{8}-\mathrm{Na}_{2} \mathrm{~S}_{2} \mathrm{O}_{3}$ & 39.60 & 37.86 \\
\hline
\end{tabular}

that the graft copolymer initiated by $\mathrm{K}_{2} \mathrm{~S}_{2} \mathrm{O}_{8}-\mathrm{Na}_{2} \mathrm{~S}_{2} \mathrm{O}_{3}$ has the largest yield and highest intrinsic viscosity, which means this binary-initiating system is more effective in grafting $\mathrm{AM}$ onto EH-lignin. For this reason, $\mathrm{K}_{2} \mathrm{~S}_{2} \mathrm{O}_{8}-\mathrm{Na}_{2} \mathrm{~S}_{2} \mathrm{O}_{3}$ was employed in our further research on the graft copolymerization of EH-lignin with AM.

\subsubsection{Effect of Initiator Dosage on Graft Copolymerization}

The effect of initiator dosage on graft copolymerization was evaluated considering the yield of copolymer and the grafting ratio. The results were shown in Fig. 2. It was found that the yield of the copolymer and the grafting ratio increased with increasing initiator dosage at first. However, when the initiator dosage was more than $3 \%$ of the weight of lignin, $\mathrm{Y}(\%)$ and GR( $\%)$ increased slowly and then decreased when the dosage of initiator reach $5 \%$.

In the process of the copolymerization, the binaryinitiating system of $\mathrm{K}_{2} \mathrm{~S}_{2} \mathrm{O}_{8}-\mathrm{Na}_{2} \mathrm{~S}_{2} \mathrm{O}_{3}$ generated free radicals to initiate the polymerization of PAM and the graft copolymerization of EH-lignin with AM or with PAM chains. On one hand, high free radical concentration may enhance the possibility of graft copolymerization and increase the yield and molecular weight of copolymer; on the other hand, it will lower the polymerization degree of PAM that may graft onto EH-lignin and decrease the molecular size of copolymer. Therefore, $\mathrm{Y}(\%)$ and $\mathrm{GR}(\%)$ reach their maximum when the contradiction reaches a equilibrium and the optimum dosage of initiator is $3 \%$ of the weight of EH-lignin.

\subsubsection{Effect of Acrylamide Dosage on Graft Copolymerization}

The mass ratio of acrylamide to EH-lignin is another important factor that may affect the yield of copolymer and the grafting ratio. It can be found in Fig. 3 that when the dosage of AM was no more than $6 \mathrm{~g}$, the grafting ratio of EH-lignin/AM copolymer increased quickly

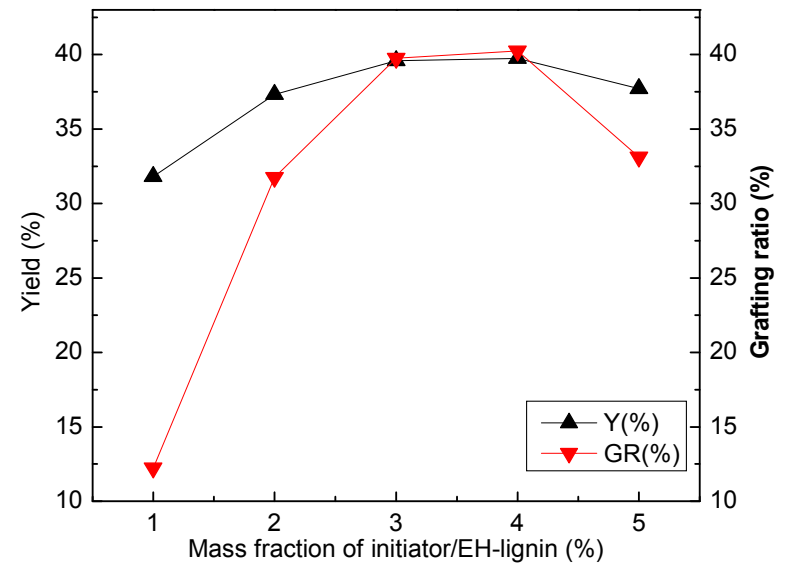

Figure 2. Effect of initiator dosage on the yield of copolymer and the grafting ratio. 


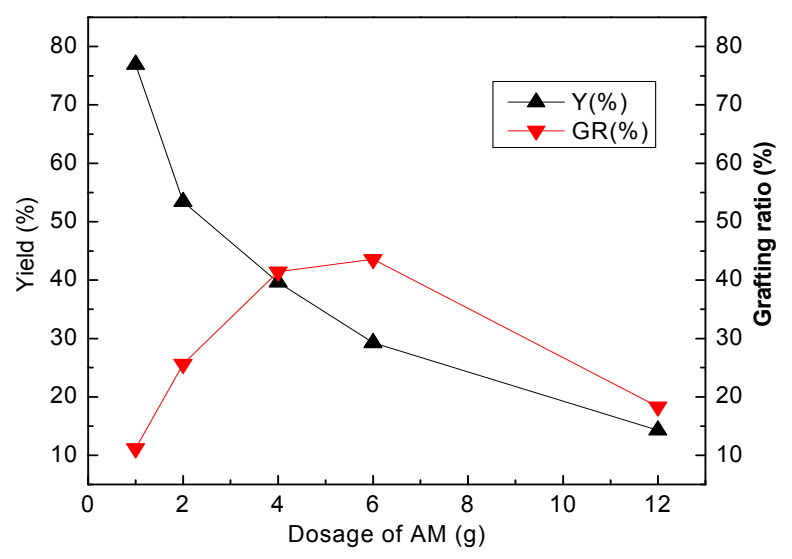

Figure 3. Effect of AM dosage on the yield of copolymer and the grafting ratio (the dosage of EH-lignin is $2 \mathrm{~g}$ ).

as the mass ratio of AM to EH-lignin increased. This is because the higher AM concentration can make it easier for EH-lignin to come into contact with monomer and then speed up graft copolymerization and improve grafting ratio. However, when the concentration of the $\mathrm{AM}$ is higher than a certain level, the probability of the homopolymerization of AM will increase rapidly. This reaction, which leads to the formation of PAM, will compete with graft copolymerization and diminish the grafting ratio. The yield of copolymer, on the contrary, decreased gradually as the dosage of AM increased from $1 \mathrm{~g}$ to $12 \mathrm{~g}$. This phenomenon can be ascribed to the rising water-solubility of the copolymer. It has been mentioned above that higher monomer concentration will enhance the probability of homopolymerization of AM and extend the length of some PAM chains that have been grafted onto EH-lignin. Thus, the water-solubility of EH-lignin/ AM copolymer increases with the rise of AM dosage and the quantity of copolymer that can be separated from aqueous solution declines simultaneously. We can see from the above analysis that the ideal dosage of acrylamide is $4 \sim 6 \mathrm{~g}$ or the mass ratio of AM to EH-lignin is 2 3.

\subsubsection{Effects of Reaction Time and Reaction Temperature on Graft Copolymerization}

The effects of reaction time and reaction temperature on the yield of copolymer and the grafting ratio were shown in Fig. 4 and Fig. 5 respectively. From Fig. 4, we could see the yield and grafting ratio improved rapidly as the reaction time increased from $2 \mathrm{~h}$ to $4 \mathrm{~h}$. When the reaction time was further prolonged, the growth of yield and grafting ratio became unremarkable. This phenomenon is similar to the regular pattern of radical polymerization. The graft copolymerization took place mostly in the period of initiation and the speed of the copolymerization was high at first. However, as the concentration of acrylamide and initiators declined with elapsed time, the graft copolymerization slowed down and the yield and grafting ratio stopped growing.

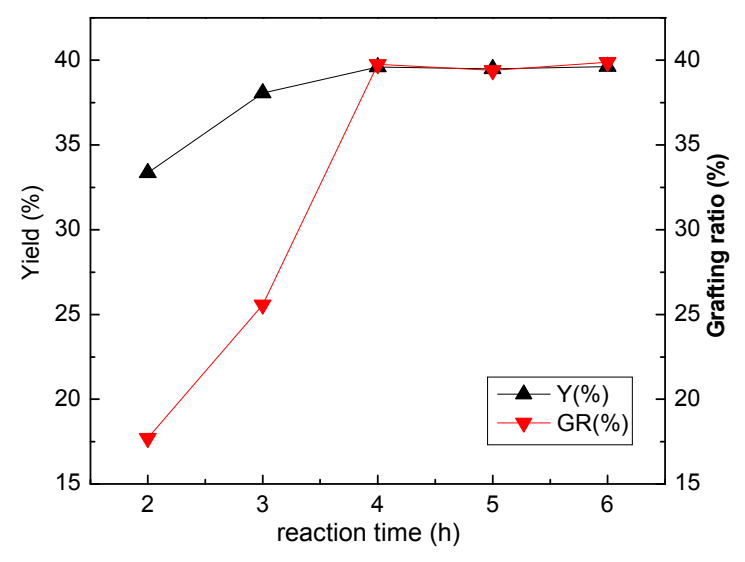

Figure 4. Effect of reaction time on graft copolymerization.

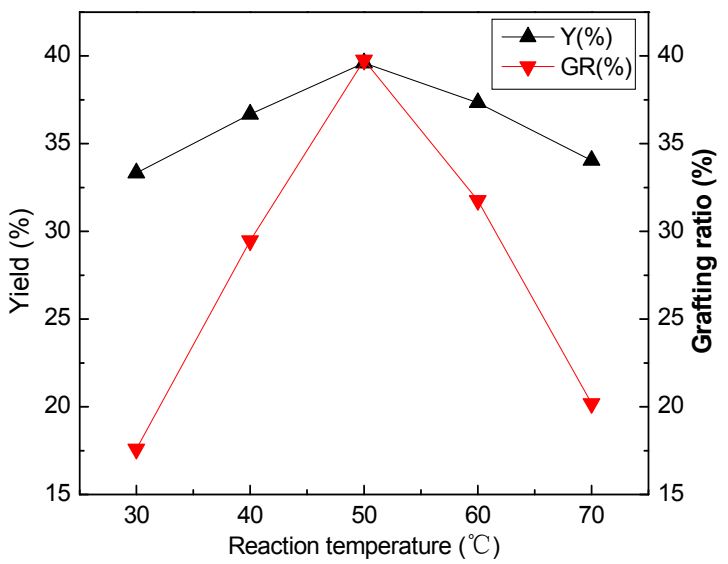

Figure 5. The influence of reaction temperature on graft copolymerization.

The influence of different reaction temperature on graft copolymerization was shown in Fig. 5. The highest yield and grafting ratio can both be reached at $50^{\circ} \mathrm{C}$ and this is the ideal reaction temperature for the binary-initiating system of $\mathrm{K}_{2} \mathrm{~S}_{2} \mathrm{O}_{8}-\mathrm{Na}_{2} \mathrm{~S}_{2} \mathrm{O}_{3}$. It is well known that low reaction temperature will postpone the decomposition of initiator and restrain the copolymerization. On the contrary, when the reaction temperature was higher than $50^{\circ} \mathrm{C}$, the decomposition of initiator would be too fast and the possibility of radical transfer would be greatly enhanced, both of which will lead to the decrease of yield and grafting ratio.

\subsection{FT-IR Spectral Analysis}

The FT-IR spectra of EH-lignin and EH-lignin/AM copolymer were shown in Fig. 6. It can be seen from the FT-IR spectrum of EH-lignin/AM copolymer, compared with pure EH-lignin, the relative intensity of the band at about $1700 \mathrm{~cm}^{-1}$ increased significantly. This adsorption peak is assigned to the vibration absorbance of $\mathrm{C}=\mathrm{O}$ and the rise of its intensity implies that AM have been grafted onto EH-lignin. Furthermore, the intensity of the 


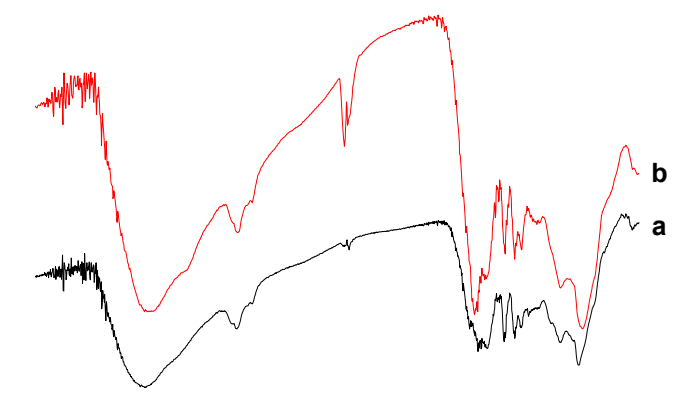

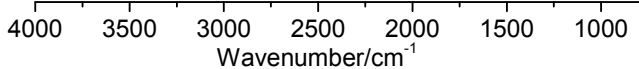

Figure 6. FT-IR spectra of EH-lignin and EH-lignin/ AM copolymer (a EH-lignin; b EH-lignin/AM copolymer).

band at $1020 \mathrm{~cm}^{-1}$, which is assigned to the absorbance of $\mathrm{N}-\mathrm{H}$, also increased and this is another proof of the successful graft copolymerization. All the information provided by FT-IR analysis had indicated that the product is an EH-lignin/AM copolymer with numerous functional groups, such as phenolic hydroxyl $\left(3400 \mathrm{~cm}^{-1}\right)$, carbonyl $\left(1700 \mathrm{~cm}^{-1}\right)$ and amide (1550 and $\left.1020 \mathrm{~cm}^{-1}\right)$ groups $[11,12]$.

\subsection{Adsorption and Decoloration of Dye- Wastewater by EH-lignin/AM Copolymer}

The adsorption and decoloration of AR 274 and RR X3B dye wastewater by EH-lignin/AM grafted copolymer and pure EH-lignin was investigated according to the procedures described in 2.3. The effect of grafting ratio on the adsorption capacity of graft copolymer was shown in Fig. 7. It was found that the absorption capacity of EH-lignin/AM copolymer to both dye wastewaters increased remarkably with the rising of grafting ratio. When the grafting ratio was $43.6 \%$, the maximum adsorption amount of AR 274 and RR X-3B by graft copolymer may reach $751 \mathrm{mg} / \mathrm{g}$ and $512 \mathrm{mg} / \mathrm{g}$ respectively.

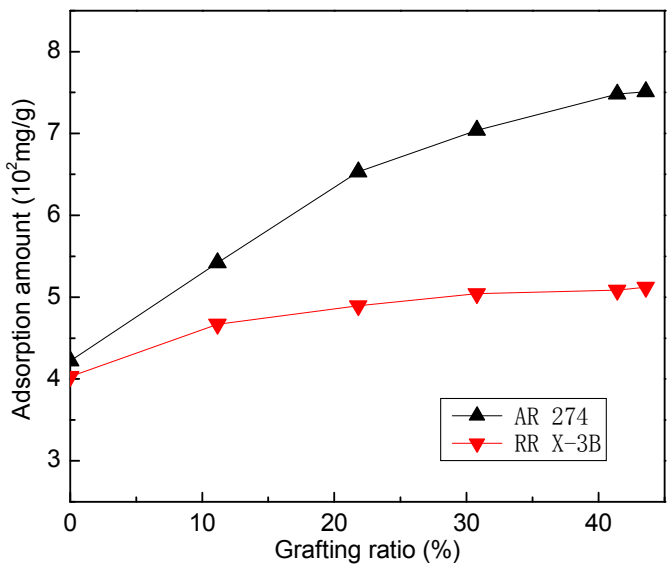

Figure 7. Effect of grafting ratio on the adsorption capacity of EH-lignin/AM copolymer.
The flocculation of dye colloids result from various mechanisms, including electrostatic attraction, sorption (related to protonated amine groups and phenolic hydroxyl), and bridging (related to the high molecular weight of the polymer) [13]. There are sulfonic, carbonyl and amino groups in AR 274 and RR X-3B. The sulfonic groups can be electrostatic attracted by protonated amide groups of the EH-lignin/AM graft copolymer and the amino groups can from hydrogen bonding with phenolic hydroxyl groups of the copolymer and EH-lignin. Thus, higher grafting ratio will help to strengthen the supramolecular interaction between dyes and graft copolymers, which can bind copolymer molecules closer, trap and flocculate dyes more effectively.

The relationship between the dosage of coagulants and the decoloration rate of dye wastewater was shown in Fig. 8. The concentration of AR 274 dye wastewater was $500 \mathrm{mg} / \mathrm{L}$ and the grafting ratio of EH-lignin/AM copolymer was $30.8 \%$. It can be seen in Fig. 8 that the decoloration rate of dye wastewater increased rapidly as the dosage of both flocculants increased from $50 \mathrm{mg} / \mathrm{L}$ to $200 \mathrm{mg} / \mathrm{L}$. When the dosage of flocculant reached $800 \mathrm{mg} / \mathrm{L}$, the AR 274 dye had almost been removed completely.

Results also showed that both pure EH-lignin and EHlignin/AM graft copolymer performed well in the removal of AR 274 from dye wastewater when the dosage of flocculant is higher than $200 \mathrm{mg} / \mathrm{L}$. As has been mentioned in 3.2, EH-lignin and lignin/AM copolymer possess lots of functional groups, such as phenolic hydroxyl, carbonyl and amide groups. Meanwhile, AR 274 also contains alcoholic hydroxyl and carbonyl groups, which leads to the adsorption of dye molecules on EH-lignin and EH-lignin/AM copolymer through hydrogen bonding. Subsequently, the flocculant molecules bridge to each other under acidic environment and form large flocs, which will trap the dyes dissolved in wastewater and then precipitate simultaneously.

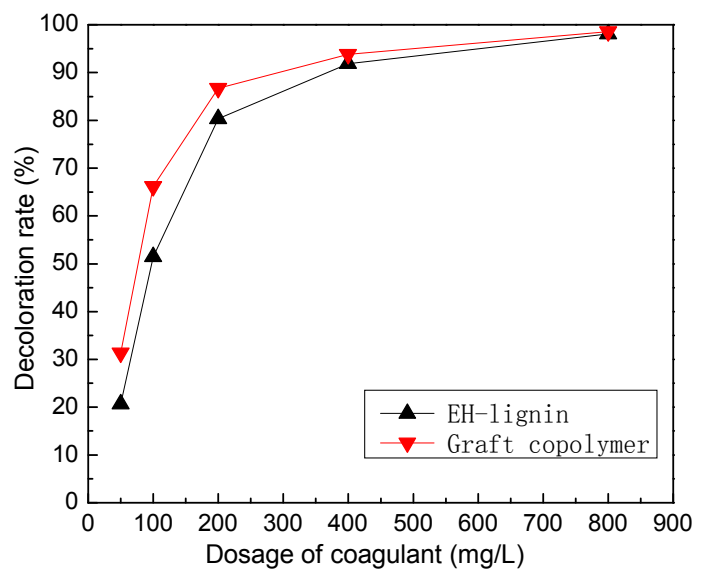

Figure 8. Effect of coagulant dosage on the decoloration rate of AR 274 wastewater. 


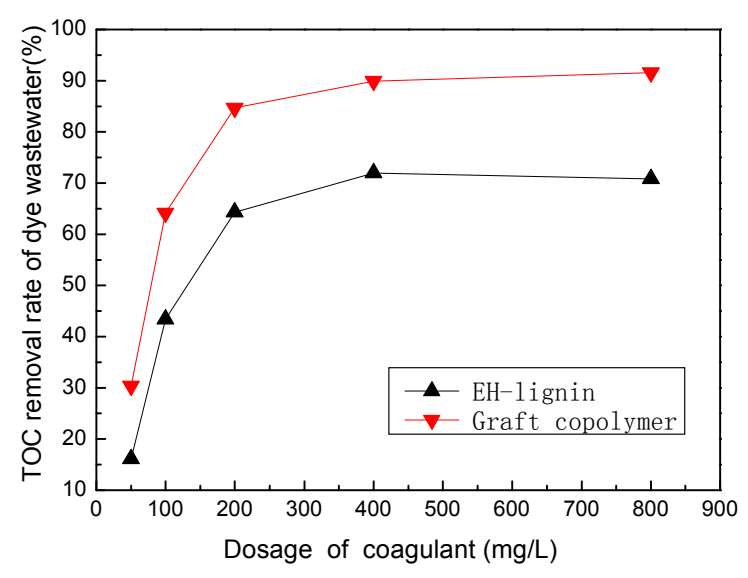

Figure 9. TOC removal rate of dye wastewater after flocculation.

The residue of EH-lignin and EH-lignin/AM copolymer in the supernatant after flocculation was measured by TOC test to evaluate the potential secondary pollution of this flocculant. These experiments were carried out with various dosage of flocculant at $\mathrm{pH}=4$. It can be found in Fig. 9 that, compared with the decoloration rate of AR 274 wastewater flocculated by both flocculants, the TOC removal rate of these samples are much lower. This phenomenon implies that part of EH-lignin and EH-lignin/AM copolymer remains in the supernatant after flocculation. Furthermore, TOC removal rate of the dye wastewater treated by EH-lignin/AM graft copolymer is much higher than that of pure EH-lignin, which means the graft copolymerization of EH-lignin with AM will help to minimize the residue amount of this copolymer in wastewater and diminish the potential secondary pollution.

\section{CONCLUSIONS}

The preparation and the application of EH-lignin/AM graft copolymer were presented in this article. The optimum synthesis conditions were discussed and the functional groups of the copolymer were characterized by FT-IR The adsorption and decoloration of two azo-dye wastewater by EH-lignin/AM grafted copolymer and pure EH-lignin was investigated.

1) The optimum conditions for the graft copolymerization of EH-lignin with acrylamide were as follows: initiator $\mathrm{K}_{2} \mathrm{~S}_{2} \mathrm{O}_{8}-\mathrm{Na}_{2} \mathrm{~S}_{2} \mathrm{O}_{3}$ with a quantity $3 \mathrm{wt} \%$ of EHlignin, mass ratio of $\mathrm{AM}$ to $\mathrm{EH}$-lignin was $2 \sim 3$, reaction time $4 \mathrm{~h}$ and temperature at $50^{\circ} \mathrm{C}$.

2) FT-IR spectrum of EH-lignin/AM copolymer indicated that acrylamide had been grafted onto EH-lignin successfully and the copolymer had numerous functional groups, such as phenolic hydroxyl, carbonyl and amide groups.

3) The absorption capacity of EH-lignin/AM copolymer to dye wastewater was enhanced with the increase of grafting ratio. Both pure EH-lignin and EH-lignin/AM graft copolymer performed well in the removal of AR 274 from dye wastewater when the dosage of flocculant is higher than $200 \mathrm{mg} / \mathrm{L}$. However, the residue concentration of EH-lignin/AM graft copolymer remained in the supernatant after flocculation was much lower than that of pure EH-lignin.

\section{ACKNOWLEDGEMENTS}

The author thanks Key Laboratory of Cellulose and Lignocellulosics Chemistry, Guangzhou Institute of Chemistry, Chinese Academy of Sciences for financial support (LCLC-2004-158).

\section{REFERENCES}

[1] Rafael, L., Lorenzo, H. D. and Juan, M. (2008) Biofuels: A technological perspective. Energy Environ. Sci., 1, 542-564.

[2] Roussy, J., Van, V. M. and Dempsey, B. A. (2005) Influence of chitosan characteristics on the coagulation and the flocculation of bentonite suspensions. Water Research, 39(14), 3247-3258.

[3] Sarasa, J., Roche, M. P. and Ormad, M. P. (1998) Treatment of a wastewater resulting from dyes manufacturing with ozone and chemical coagulation. Water Research, 32(9), 2721-2727.

[4] Bousher, A., Shen, X. and Edyvean, R. G. (1997) Removal of coloured organic matter by adsorption onto low-cost waste materials. Water Research, 31(8), 2084-2092.

[5] Wu, Y., Zhang, S. and Guo, X. (2008) Adsorption of chromium (III) on lignin. Bioresource Technology, 99(16), 7709-7715.

[6] Dizhbite, T., Zakis, G. and Kizima, A. (1999) Lignin-a useful bioresource for the production of sorption-active materials. Bioresource Technology, 67(3), 221-228.

[7] Cheng, X. S. and Liu X. L. (2006) Separation of lignin from cornstalks residue by enzymatic hydrolysis and its properties. Modern Chemical Industry, 26, Supplement, 99-102.

[8] Fang, R., Chen, Y. P. and Cheng X. S. (2007) Application of organosolv lignin on polymer synthesis and modification. Journal of Cellulose Science and Technology, 15(3), $56-60$.

[9] Zhang, X. H., Cheng, X. S. and Tang, J. (2006) Adsorption of bromelain with HBS lignin and its derivatives. Chinese Journal of Process Engineering, 6, 87-90.

[10] Chen, Y. P., Zhang, T. T. and Cheng X. S. (2005) The adsorption of phenol by high boiling solvent lignin. Journal of Cellulose Science and Technology, 13(2), 20-25.

[11] Zhao, B. Y., Hu, K. A., Fan, Y. Z., Zhu, P. F. and Wu, R. J. (2000) Infrared spectroscopic study on lignosulfonic acid and its derivatives. Chinese Journal of Analytical Chemistry, 28, 716-719.

[12] Chen, Y. P. and Cheng X. S. (2008) Preparation and characteristic analysis of rice husk high boiling solvent lignin. Journal of Forestry Research, 19, 159-163.

[13] Roussy, J., Van, V. M. and Dempsey, B. A. (2005) Influence of chitosan characteristics on the coagulation and the flocculation of bentonite suspensions. Water Research, 39(14), 3247-3258. 